Cite as: Low Temp. Phys. 35, 526 (2009); https://doi.org/10.1063/1.3168638

Published Online: 11 September 2009

A. G. Grechnev, A. S. Kovalev, and M. L. Pankratova

\section{ARTICLES YOU MAY BE INTERESTED IN}

Magnetization field-dependences and the "exchange bias" in ferro/antiferromagnetic systems. I. Model of a bilayer ferromagnetic

Low Temperature Physics 35, 476 (2009); https://doi.org/10.1063/1.3151994

LOW TEMPERATURE TECHNIQUES OPTICAL CAVITY PHYSICS MITIGATING THERMAL \& VIBRATIONAL NOISE

\section{DOWNLOAD THE WHITE PAPER}




\title{
Field dependences of the magnetization and exchange bias in ferro/antiferromagnetic systems. II. Continuum model of a ferromagnetic layer
}

\author{
A. G. Grechnev and A. S. Kovalev ${ }^{\text {a) }}$ \\ B. I. Verkin Institute for Low-Temperature Physics and Engineering of the National Academy of Sciences of \\ Ukraine, pr. Lenina 47, Kharkov 61103, Ukraine
}

\section{L. Pankratova}

V. N. Karazin Kharkov National University, pl. Svobody 4, Kharkov 61107, Ukraine

(Submitted March 16, 2009)

Fiz. Nizk. Temp. 35, 670-676 (July 2009)

A model giving a qualitative explanation of the results of an experimental investigation of the field dependences of the magnetization in ferro- and antiferromagnetic media in contact with one another is proposed. In this model a thin ferromagnetic (FM) film with strong easy-plane anisotropy is described in the continuum approximation. It is shown that collinear and canted structures of the ferromagnetic layer can co-exist in the system studied. The ranges of the parameters (exchange interactions in the FM layer and through the FM/AFM interface, thickness of the FM layer, and magnitude of the external magnetic field) where a nonuniform state of the FM exists are found and explicit analytic expressions are obtained for the distribution of the magnetization in this state. The dependences of the total magnetization of the system on the magnitude of the external field are constructed in the physically important cases of "thin" and "thick" FM layers, and it is demonstrated that the experimentally observed unsymmetric dependence $M(H)$ is possible. The results of the present work are compared with our previous results obtained using simpler model of a two-layer FM subsystem. (C) 2009 American Institute of Physics.

[DOI: $10.1063 / 1.3168638]$

\section{INTRODUCTION}

Magnetic multilayer systems with a different magnetic structure of the individual layers (primarily, alternating layers of ferro- and antiferromagnets) have been attracting the attention of experimentalists and theoreticians recently. This is due to the possible applications of such objects in the magnetic writing and storage of information and in the study and application of the phenomenon of giant magnetoresistance. The exchange bias (EB) phenomenon or exchange anisotropy, ${ }^{1-3}$ consisting of a shift of the hysteresis loop of the dependence of the magnetization on the external magnetic field $M=M(H)$ away from the symmetric position relative to the point $H=0$, is very interesting from the theoretical standpoint. The effect is of a surface character and is determined by the magnetic interaction through the FM/AFM interface surface, and the magnitude of the EB field $H_{\text {bias }}$ decreases with increasing thickness of the FM layer as $H_{\text {bias }}$ $\sim 1 / L_{F}$, where $L_{F}$ is the thickness of the FM layer. ${ }^{2,3}$ The simplest theoretical models of the phenomenon (for example, Ref. 2) assume that the magnetization of the FM layer is uniform and absence of nonuniform magnetic states. Exchange bias is explained by the effective field acting on the FM through the interface. It is assumed that the AFM boundary is compensated, though the EB phenomenon is also observed in cases where the boundaries are not compensated. The possibility of the formation of domain walls (DWs), parallel to the interface was examined in Refs. 4-10, but even though many works have been performed a complete theory of EB still does not exist.
Recently, new features of the EB phenomenon have been found experimentally: the shifted hysteresis loop becomes asymmetric (i.e. $\left.M\left(2 H_{\text {bias }}-H\right) \neq-M(H)\right)$ and steps appear on it, ${ }^{11-15}$ and the slope of the magnetization curve is different on different sections. The presence of "small shelves" and sections of the function $M(H)$ which are separated by field intervals indicates the possible existence of stationary states which are different from completely magnetically reversed states.

In Ref. 16 we examined the simplest model of a FM/ AFM bilayer system where the FM part consisted of only two atomic layers. This was the simplest model that admits nonuniform AFM states of magnetization in a direction perpendicular to the FM/AFM interface.

It was shown that (i) because of the formation of a noncollinear (canted) phase the region of magnetization reversal is of finite width as a function of the field, (ii) for ferromagnetic exchange $J$ which is small compared with the exchange $J_{0}\left(J<J_{0} / 2\right)$ through the interface a "small shelf" with $M$ $=0$, corresponding to the antisymmetric phase $\uparrow \downarrow$, is observed in the field dependence of the magnetization of the ferromagnetic layer near the field $H_{\text {bias }}$, (iii) the dependence $M(H)$ is symmetric relative to the field $H_{\text {bias, }}$, and (iv) FM magnetic anisotropy in the easy plane results in the appearance of a strongly asymmetric hysteresis loop. The results describe qualitatively a number of experimentally observed dependences of the magnetization curves but they do not describe, for example, the asymmetry of the magnetization curves with respect to the value of $M$.

Our objective in the present work is to extend the previously proposed model to the case of a FM region consisting 
of a quite large number of magnetic layers. We show that such an extension of the model results in asymmetry of $M(H)$ associated with the appearance of DW type nonuniform states. In addition, it is demonstrated that the results in the bi- and multilayer models behave similarly but an increase of the number of FM layers increases the critical value of the parameter $J / J_{0}$ at which the special features of the EB phenomenon appear. This result can serve as validation of the model examined in Ref. 16 and of the results following from this model.

\section{FORMULATION OF THE CONTINUUM MODEL OF A FM LAYER OF FINITE THICKNESS}

The model of two FM layers which was studied in Ref. 16 is very simplified, but it does yield some important qualitative results. Actually, in the experiment the thickness of the ferromagnetic part reaches several tens of atomic layers, and then it is sometimes possible to use continuum models. We shall consider the case where the FM consists of $N$ layers (Fig. 1). Just as in Ref. 16, we shall confine our attention to a "rigid" AFM whose magnetic moments are fixed and do not change direction in an external magnetic field. The strong easy-plane anisotropy "packs" the FM magnetic moments into the easy plane, and the configuration of the magnetic moments is characterized only by the turn angles $\varphi_{n}$ of the moments in the this plane. We shall examine the simplest case where there is no additional anisotropy in the easy plane (isotropic easy plane). The parameter $J$ characterizes the exchange interaction between the FM moments, and the parameter $J_{0}$ characterizes the exchange interaction through the FM/AFM interface. The total energy of the ferromagnetic part is given by the expression

$$
E=-J_{0} \cos \varphi_{1}-J \sum_{n=1}^{N-1} \cos \left(\varphi_{n}-\varphi_{n+1}\right)-H \sum_{n=1}^{N} \cos \varphi_{n},
$$

where $H$ is the external magnetic field, directed along the interface of the media.

The equilibrium configurations are determined by the equations $d E / d \varphi_{n}=0$ for all $n=1 \ldots, N$. These equations have the form

$$
\begin{aligned}
& \left(H+J_{0}\right) \sin \varphi_{1}+J \sin \left(\varphi_{1}-\varphi_{2}\right)=0, \\
& H \sin \varphi_{n}+J \sin \left(\varphi_{n}-\varphi_{n-1}\right)+J \sin \left(\varphi_{n}-\varphi_{n+1}\right)=0, \\
& n=2, \ldots, N-1, \\
& H \sin \varphi_{N}+J \sin \left(\varphi_{N}-\varphi_{N-1}\right)=0 .
\end{aligned}
$$

For weak external fields $|H| \ll J$ and weak effective exchange through the interface $J_{0} \ll J$ (for sufficiently thin FM

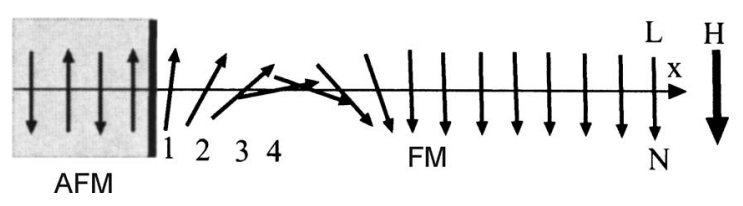

FIG. 1. Configuration of the magnetic moments of FM layers that corresponds to the continuum model studied in the present work. layer), the continuum approximation can be used and the system can be described by means of differential equations. We shall switch from discrete variables $\varphi_{n}$ to a continuous function $\varphi(x)$, where $0<x<L, L=a N$ is the thickness of the ferromagnetic layer and $a$ is the interlayer distance. The equation assumes the form of the equation for a mathematical pendulum or static reduction to the "sinusoidal Gordon equation" well-known in nonlinear mechanics:

$$
\frac{d^{2} \varphi}{d x^{2}}=\frac{H}{J a^{2}} \sin \varphi
$$

This equation is supplemented by boundary conditions at the boundary with the antiferromagnet at $x=0$ and at the free boundary at $x=L$ :

$$
\left.\frac{d \varphi}{d x}\right|_{x=0}=\frac{J_{0}}{a J} \sin \varphi(0),\left.\quad \frac{d \varphi}{d x}\right|_{x=L}=0
$$

\section{NONUNIFORM STATES OF A FM LAYER}

The only solution of the system (5) and (6) for $H>0$ is the uniform state $\varphi=0$. Non-collinear solutions exists for $H$ $<0$, where a large restructuring of the ferromagnetic layer occurs. We introduce the notation $\mathcal{H}=|H| / J a^{2}$ and rewrite Eq. (5) in the form

$$
\frac{d^{2} \varphi}{d x^{2}}+\mathcal{H} \sin \varphi(x)=0 .
$$

This equation possesses the trivial solution: $\varphi=0$ with energy $\mathcal{E}_{\uparrow}=L \mathcal{H} / a-\mathcal{H}_{0}$ and $\varphi=\pi$ with $\mathcal{E}_{\downarrow}=L \mathcal{H} / a+\mathcal{H}_{0}$, where the total energy is determined in the natural manner

$$
\mathcal{E}=\frac{1}{a} \int_{0}^{L} d x\left\{\frac{1}{2}\left(\frac{d \varphi}{d x}\right)^{2}+\mathcal{H}_{0} \cos \varphi\right\}-\mathcal{H}_{0} \cos \varphi_{0}-\mathcal{H} \frac{L}{a},
$$

and $\mathcal{H}_{0}=J_{0} / J a^{2}$. In addition to these collinear solutions there exist solutions of Eq. (7) which correspond to a canted phase:

$$
\varphi(x)=2 \arcsin \left[k \operatorname{sn}\left(\sqrt{\mathcal{H}}\left(x+x_{0}\right), k\right)\right],
$$

satisfying the boundary conditions, where sn $(q, k)$ is Jacobi's elliptic sine and $k$ is its modulus. The parameters $\left(x_{0}, k\right)$ of this solution are found from the boundary conditions (6). They are determined by the following system of equations:

$$
\begin{aligned}
& \sqrt{\lambda} \operatorname{cn}\left(\sqrt{\mathcal{H}} x_{0}, k\right)=\operatorname{sn}\left(\sqrt{\mathcal{H}} x_{0}, k\right) \operatorname{dn}\left(\sqrt{\mathcal{H}} x_{0}, k\right), \\
& \sqrt{\mathcal{H}}\left(L+x_{0}\right)=\mathbf{K}(k),
\end{aligned}
$$

where $\mathbf{K}(k)$ is the complete elliptic integral of the first kind and $\lambda=J|H| / J_{0}^{2}$. The system of equations (10) and (11) reduces to a single equation for the modulus $k$ of the elliptic function:

$$
\operatorname{sn}(\sqrt{\mathcal{H}} L, k) \operatorname{dn}(\sqrt{\mathcal{H}} L, k)=\sqrt{\lambda} \operatorname{cn}(\sqrt{\mathcal{H}} L, k)
$$

This equation contains two dimensionless parameters: $\lambda$ and $\sqrt{\mathcal{H}} L=\sqrt{|H| / J}(L / a)$. Three parameters with the dimension of length arise naturally: $L, l_{0}=a\left(J / J_{0}\right)$, and $l_{1}$ $=a \sqrt{J /|H|}$. Here $\lambda=\left(l_{1} / l_{0}\right)^{2}$ and $\sqrt{\mathcal{H}} L=L / l_{1}$. We call attention to the following relation between the arguments of the ellip- 


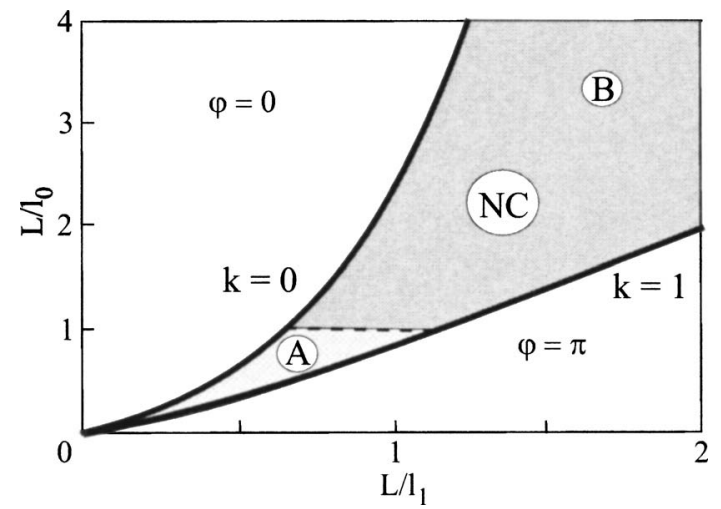

FIG. 2. Phase diagram in the plane of the parameters $L / l_{1}$ and $L / l_{0}$. The regions of stability of the collinear phases with $\varphi=0$ and $\varphi=\pi$ and the canted phase (NC) are presented. The regions $A$ and $B$ correspond to "thin" $\left(L \ll L_{0}\right)$ and "thick" $\left(L \gg l_{0}\right)$ FM layers.

tic functions: $\sqrt{\lambda} \operatorname{sn}\left(\sqrt{\mathcal{H}} x_{0}, k\right)=\operatorname{sn}(\sqrt{\mathcal{H}} L, k)$. The energy of the canted configuration (9) equals (in dimensionless units)

$$
\begin{aligned}
a^{2} E_{N C}= & \frac{a L}{l_{1}^{2}}\left(2 k^{2}-3\right)-\frac{a}{l_{0}}+\frac{a l_{0}}{l_{1}^{2}} 2 k^{2} \operatorname{sn}^{2}\left(\frac{L}{l_{1}}, k\right) \\
& +4 \frac{a}{l_{1}}\left[\mathbf{E}(k)-E\left\{\arcsin \frac{l_{0}}{l_{1}} \operatorname{sn}\left(\frac{L}{l_{1}}, k\right), k\right\}\right],
\end{aligned}
$$

where $\mathbf{E}(k)$ and $E(u, k)$ are the complete and incomplete elliptic integrals of the second kind, and Eq. (12) can be rewritten in the form

$$
\operatorname{sn}\left(\frac{L}{l_{1}}, k\right)=\frac{1}{\sqrt{2} k} \sqrt{1+\lambda-\sqrt{(1-\lambda)^{2}+4 \lambda\left(1-k^{2}\right)}} .
$$

Using Eq. (13) the energy of the canted configuration can be expressed in terms of the modulus $k$ of the elliptic function, and the expression (14) is an equation for the dependence of this modulus on the magnitude of the external magnetic field $H$ for different ratios of the parameters of the system. In the expression (14) the parameter $\lambda$ can be arbitrary (larger or smaller than 1); this follows from the relation for $\operatorname{dn}^{2}\left(L / l_{1}, k\right)$.

We shall also calculate the total magnetization of the ferromagnetic layer, given by the relation

$$
M=\frac{1}{J a^{3}} \int_{0}^{L} d x \cos \varphi(x) .
$$

The normalization is chosen so that the natural relation $E=-M H$ holds in the collinear phase. In the collinear phases $M_{\uparrow}=L / J a^{3}$ and $M_{\downarrow}=-L / J a^{3}$. The magnetization of the canted phase of the ferromagnetic film is given by (the ratios of the characteristic parameters with the dimension of length are written out explicitly)

$$
J a^{2} M_{N C}=-\frac{L}{a}+2 \frac{l_{1}}{a}\left[\mathbf{E}(k)-E\left\{\arcsin \frac{l_{0}}{l_{1}} \operatorname{sn}\left(\frac{L}{l_{1}}, k\right), k\right\}\right] .
$$

The equation (14) is easily studied in the plane of the parameters $L / l_{1}$ and $L / l_{0}$ (Fig. 2). In this figure the lines $k$ $=0$ and $k=1$ bound the region of existence of different solutions. The lower boundary of the region of the canted phase

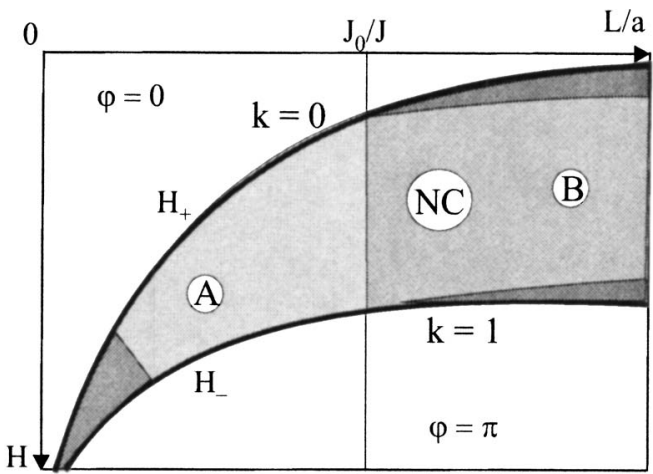

FIG. 3. Phase diagram in the plane $|H|$ and $L / a$. The lines $H_{ \pm}$correspond to the boundaries of the region of existence of the canted phase in Fig. 4. The line $L / a=J_{0} / J$ separates the regions of "thin" $(A)$ and "thick" $(B)$ FM layers. The dark regions are regions where the inequalities used to find explicit dependences for the parameters of the magnetic structure on the magnitude of the field and the thickness of the FM layer are satisfied.

$\left(H=H_{-}\right)$in the figure is determined by the relation $L / l_{0}$ $=\left(L / l_{1}\right) \tanh \left(L / l_{1}\right)$ and the upper boundary $\left(H=H_{+}\right)$by the relation $L / l_{0}=\left(L / l_{1}\right) \tan \left(L / l_{1}\right)$. In the present article we examine the case of a quite thick FM interlayer: $L / a \gg 1$. In addition, the physical situation corresponds to the values $J$ $\gg J_{0}$ and therefore $l_{0} / a \gg 1$. Thus there are two large parameters in the problem at hand: $L / a$ and $l_{0} / a$. The case $L \gg l_{0}$ corresponds to a "thick" ferromagnetic layer (region $B$ in Fig. 2) and $L \ll l_{0}$ to a "thin" ferromagnetic layer (region $A$ ). In the figure, the line $L=l_{0}$ corresponds to the boundary of these limiting cases. The two cases indicated were studied qualitatively in Ref. 9, but the exact solutions of the model are obtained for the first time in the present article. The solution of Eq. (14) $k=k(H)$ is easily found within the "thin" and "thick" FM layers, determining the change of the structure of the canted state as a function of the field strength. In the region $A$ of small values of $l_{1} / l_{0}$ ("thin" FM layer) we have $2 k^{2}(H) \approx 1+3\left(J / J_{0}\right)\left[H / J_{0}+(L / a)\left(H / J_{0}\right)^{2}\right]$. In the region $B$ of large values of $l_{1} / l_{0}$ ("thick" FM layer) we have $(k)=\mathbf{K}\left(L / a+J / J_{0}\right) \sqrt{|H| / J}$.

Since we shall be interested below in the dependences of the energy and magnetization of the canted state on the external field, we shall find the field interval in which this state exists as a function of the parameter ratios $L / a$ and $J / J_{0}$. This region is depicted in Fig. 3 as a function of the parameter $L / a$ for fixed ratio $J / J_{0}$. The region of existence of the canted configuration is singled out; its lower field boundary corresponds to the modulus $k=0$ and the upper field boundary to $k=1$. It is evident in the figure that a canted structure exists in a "thin" FM film only in strong fields $\left(|H| \gg J_{0}\right)$ and in the "thick" film only in weak fields $\left(|H| \ll J_{0}\right)$.

\section{FIELD DEPENDENCES OF THE MAGNETIZATION IN THE CONTINUUM MODEL}

We shall examine first the case of a "thin" FM layer $\left(L \ll l_{0}\right)$. This case most often arises in experiments, since the typical thickness of the FM film is 10-50 atomic layers, while the effective parameter $J_{0} / J$ is of the order of $10^{-2}-10^{-3}$. We obtain from the relations presented above for the boundaries of the region of existence of the canted phase 

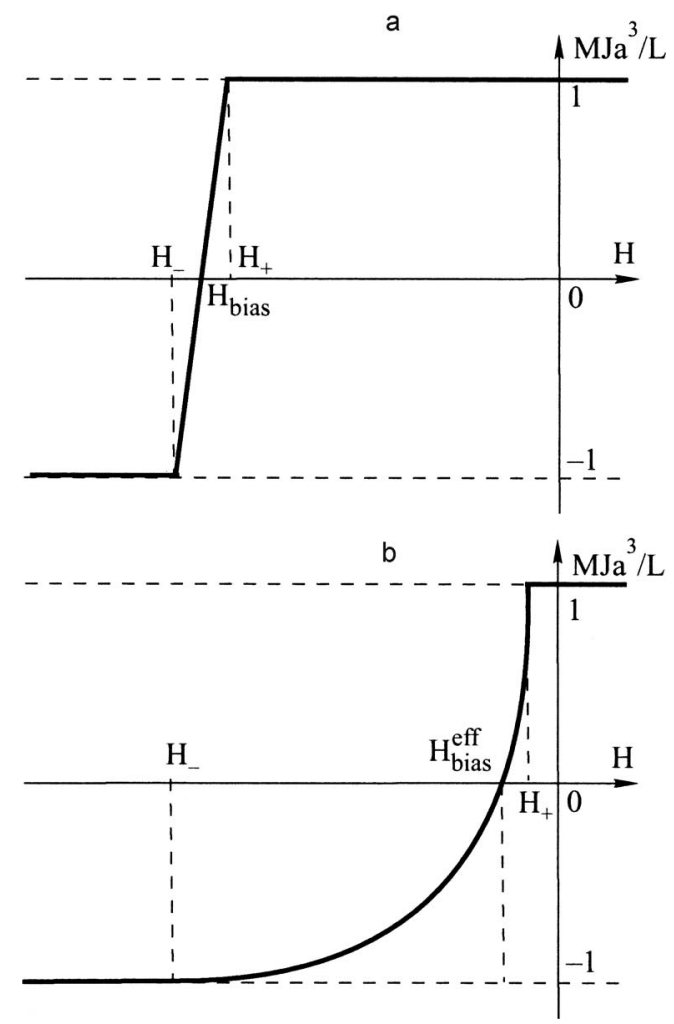

FIG. 4. Field dependence of the magnetization $M(H)$ for "thin" (a) and "thick" (b) FM layers.

$$
H_{ \pm} \approx-J_{0} \frac{a}{L} \pm \frac{J_{0}^{2}}{3 J}
$$

The field dependence of the magnetization $M(H)$ for a "thin" FM layer is presented in Fig. 4a. It is reminiscent of the corresponding dependence in the model of two FM layers, presented in Fig. 4a of Ref. 16. The transition is sharp and practically symmetric relative to the exchange bias field

$$
H_{\text {bias }} \approx-J_{0} \frac{a}{L} .
$$

This result is identical to that obtained in the simple single-layer model proposed in Ref. 1. The relation $\left|H_{\text {bias }}\right|$ $\sim 1 / L$, which is usually observed in experiments, holds here.

The width of the region of the canted phase is $\Delta H$ $=(2 / 3) J_{0}^{2} / J \ll\left|H_{\text {bias }}\right|$. For $H=H_{\text {bias }}$ the magnetization is 0 and $k^{2}=1 / 2$. Physically, the "thin" FM layer situation signifies that the FM in the canted phase is magnetized almost uniformly: $\varphi(0) \approx \varphi(L)$. Indeed, the coordinate $x$ enters in the relation (9) only in the combination $\sqrt{\mathcal{H}} x$, and $\sqrt{\mathcal{H}} L=L / l_{1}$ $\approx \sqrt{L / l_{0}}$, since in this limit $H \approx-J_{0} a / L$ in the canted phase. Therefore $\sqrt{\mathcal{H}} L \ll 1$. Practically uniform rotation of the magnetization from $\varphi=0$ to $\varphi=\pi$ occurs in the narrow region $H_{-}<H<H_{+}$.

In the bilayer model studied in Ref. $16 L=2 a$, and the "small thickness" of the layer $L=2 a \ll l_{0}$ meant that $J / J_{0}$ $\gg 1$, i.e. it corresponded to the limit $I \gg 1$ in Fig. 2a of Ref. 16. Interestingly, even though the bilayer model is simple, it gives a result that is correct not only qualitatively but also quantitatively in the limit studied. Indeed, setting $L=2 a$ in the relations (17) and (18) an expression is obtained for the center of the transitional region $H_{\text {bias }}=-J_{0} / 2$, which is iden- tical to the result obtained in the bilayer model. The width of the transitional region $\Delta H=(2 / 3) J_{0}^{2} / J$ likewise is practically identical to that obtained in the preceding model $\Delta H$ $=(1 / 4) J_{0}^{2} / J$.

We shall now examine the other limiting case-a "thick" ferromagnetic layer, when $L \gg l_{0}$. The limits $k \rightarrow 0$ and $k \rightarrow 1$ are also easily analyzed in this case. The boundaries of the canted phase in Fig. 3 are determined by the expressions

$$
H_{+} \approx-J\left(\frac{\pi}{2}\right)^{2}\left(\frac{a}{L}\right)^{2}, \quad H_{-} \approx-J\left(\frac{J_{0}}{J}\right)^{2} .
$$

Figure 3 displays the regions where the inequalities $l_{1} / l_{0}$, $L / l_{1} \sim \sqrt{L} \ll 1$ hold for a "thin" FM layer with $L / a \ll J / J_{0}$ and the regions $L / l_{1} \gg 1$ and $l_{1} / l_{0} \gg 1$ at the upper and lower boundaries with $L / a \gg J / J_{0}$ for the "thick" FM layer.

The field dependence of the magnetization $M(H)$ is unsymmetric for a "thick" FM layer (Fig. 4b) as a function of the field relative to the value $H_{0}=J_{0} a / L$. We introduce the notation $H_{\text {bias }}^{\text {eff }}$ for the field in which the magnetization vanishes, where now $\left|H_{\text {bias }}^{\text {eff }}\right| \approx\left|H_{+}\right| \approx J(a / L)^{2} \ll J_{0} a / L$ and $a^{2} J M_{N C}\left(H_{0}\right)=-(L / a)+O\left(J / J_{0}\right)$. Thus we have $\left|H_{\text {bias }}^{\text {eff }}\right| \sim 1 / L$ in this case. The simple model used in Ref. 1 no longer works in the limit considered. We obtained the decrease of the exchange bias field observed experimentally in thick samples (in Ref. 17) as a function of sample thickness $\left|H_{\text {bias }}\right| \sim 1 / L^{2}$ instead of $\left|H_{\text {bias }}\right| \sim 1 / L$ for thin FM films. The width of the transition is $\Delta H \approx J_{0}^{2} / J \gg\left|H_{\text {bias }}^{\text {eff }}\right|$. Qualitatively, the reason for the asymmetry of the magnetization reversal lies in the following for the thick region of the FM. In the collinear phase $\varphi=\pi$ there is an energy loss $2 J_{0}$ at the interface. Consequently, the formation of a nonuniform structure with width of the order of $\mathcal{H}^{-1 / 2}=l_{1}=a \sqrt{J /|H|}$ and energy $\sim \sqrt{|H| J}$ is energetically more advantageous in not very strong fields. Such an object is mathematically similar to a domain boundary. However, in our case the anisotropy in the easy plane is absent and the external magnetic field plays the role of this anisotropy in the formation of a nonuniform structure. For $H=H_{-}=-J_{0}^{2} / J$ the energy of the nonuniform state becomes of the order of $J_{0}$ and a transition occurs into the collinear phase with $\varphi=\pi$.

We note that in the model of a bilayer FM subsystem examined in Ref. 16 the limit $J_{0} \gg J$ formally corresponds to the case of a wide FM layer. That is, the limit $J / J_{0} \ll 1$, which seems to be physically groundless in the bilayer model, corresponds in the case of a FM layer of finite thickness to the entirely physical situation $L / a \gg J / J_{0}$, which can happen for $J / J_{0} \gg 1$. However, in contrast to a thin FM, in the limit considered the two models give a very different picture of magnetization reversal in a field.

\section{CONCLUSION}

Using a continuum model of a FM layer in contact with a rigid AFM film an exact solution for the canted phase was found in terms of Jacobi's elliptic functions and the boundaries within which such a phase exists were determined. Exact expressions were found for the dependences of the energy and magnetization of the system on the magnitude of the external field. The limiting cases of "thin" $\left(L / a \ll J / J_{0}\right)$ and "thick" $\left(L / a \gg J / J_{0}\right)$ FM layers were analyzed. In the case of a "thin" FM the magnetization in the canted phase is 
practically uniform and the exchange bias field is determined by the usual expression $H_{\text {bias }}=-J_{0} a / L$ (Ref. 1) and depends on the thickness of the FM layer as $\left|H_{\text {bias }}\right| \sim 1 / L$. The magnetization reversal process consists in turning of the magnetization in narrow interval of fields $\Delta H \ll\left|H_{\text {bias }}\right|$, and the magnetization curve is practically symmetric relative to $H_{\text {bias. }}$.

In the case of a "thick" FM $\left(L / a \gg J / J_{0}\right)$ the magnetization reversal process is sharply asymmetric and occurs in wide interval of fields $\Delta H \gg\left|H_{\text {bias }}^{\text {eff }}\right|$. The exchange bias field depends on the thickness of the FM layer as $\left|H_{\text {bias }}^{\text {eff }}\right| \sim 1 / L^{2}$. The reason for the asymmetry of the transition is that a domain wall type transitional region forms near the interface. We underscore that the asymmetry of the magnetization curve $M(H)$ arises in the quite simple model of the EB phenomenon without making the additional assumption, as done in Refs. 11-13, that the external field makes a finite angle with the direction of the magnetic moments of the AFM subsystem.

Partial financial support for this work was provided by a contract for collaboration between the National Academy of Sciences of Ukraine (Ukraine) and CNRS (France), a grant from the National Academy of Sciences of Ukraine for research on nanostructures, a grant for scientific collaboration between the National Academy of Sciences of Ukraine and RFFI (Russia) N 8-2009, a grant from the Royal Society of UK (Great Britain), and a grant from the Swedish Academy of Sciences.
a)E-Mail: kovalev@ilt.kharkov.ua

${ }^{1}$ W. H. Meiklejohn and C. P. Bean, Phys. Rev. 102, 1413 (1956).

${ }^{2}$ J. Nogués and I. K. Schuller, J. Magn. Magn. Mater. 192, 203 (1999).

${ }^{3}$ A. E. Berkowitz and K. Takano, J. Magn. Magn. Mater. 200, 552 (1999).

${ }^{4}$ L. Neel, Ann. Phys. (Paris) 2, 61 (1997).

${ }^{5}$ D. Mauri, H. C. Siegmann, P. S. Bagus, and E. Kay, J. Appl. Phys. 62, 3047 (1987).

${ }^{6}$ M. Kiwi, J. Mejfa-López, R. D. Portugal, and R. Ramírez, Appl. Phys. Lett. 75, 2995 (1999).

${ }^{7}$ M. Kiwi, J. Mejfa-López, R. D. Portugal, and R. Ramírez, Europhys. Lett. 48, 573 (1999).

${ }^{8}$ M. Kiwi, J. Mejfa-López, R. D. Portugal, and R. Ramírez, Solid State Commun. 116, 315 (2000).

${ }^{9}$ J. Mejfa-López, R. Ramírez, and M. Kiwi, J. Magn. Magn. Mater. 241, 346 (2002).

${ }^{10}$ M. D. Stiles and R. D. McMichael, Phys. Rev. B 59, 3722 (1999).

${ }^{11}$ M. R. Fitzsimmons, P. Yasliar, C. Leighton, J. Nogués, J. Dura, C. F. Majkrzak, and I. K. Schuller, Phys. Rev. Lett. 84, 3986 (2000).

${ }^{12}$ M. Gierlings, M. J. Prandolini, H. Fritzshe, M. Gruyters, and D. Riegel, Phys. Rev. B 65, 092407 (2002).

${ }^{13}$ A. Hoffmann, Phys. Rev. Lett. 93, 097203 (2004).

${ }^{14}$ D. N. Merenkov, A. N. Bludov, S. L. Gnatchenko, M. Baran, R. Szymczak, and V. A. Novosad, Fiz. Nizk. Temp. 33, 1260 (2007) [Low Temp. Phys. 33, 957 (2007)].

${ }^{15}$ S. L. Gnatchenko, D. N. Merenkov, A. N. Bludov, V. V. Pishko, Yu. A. Shakhayeva, M. Baran, R. Szymczak, and V. A. Novosad, J. Magn. Magn. Mater. 307, 263 (2006).

${ }^{16}$ A. G. Grechnev, A. S. Kovalev, and M. L. Pankratova, Fiz. Nizk. Temp. 35, 603 (2009) [Low Temp. Phys. 35, No. 6 (2009)].

${ }^{17}$ E. Goto, N. Hayashi, and T. Miyashita, J. Appl. Phys. 36, 2951 (1965).

Translated by M. E. Alferieff 